\title{
Pulmonary pathology of COVID-19
}

\begin{abstract}
Currently, animal-to-human transmission of SARS-CoV-2 (COVID-19) has not yet been confirmed, whereas the main mode of transmission is human-to-human. Droplets are the main route of human-to-human transmission, whereas aerosols could be another route in addition to stool-based transmission. Currently, no evidence is available to indicate intrauterine vertical transmission of SARS-CoV-2 (COVID-19) in pregnant women. In the host, the life cycle of coronavirus consists of 5 steps: 1) attachment, 2) penetration, 3) biosynthesis, 4) maturation, and 5) release. Once viruses bind to host receptors (attachment), they enter host cells, particularly type II pneumocytes via endocytosis or membrane fusion (penetration). Once viral contents are released inside the host cells, viral RNA enters the host's nucleus for replication and making viral proteins (biosynthesis). New viral particles are produced (maturation) and released. Spike protein of coronaviruses which determines the diversity of coronaviruses and host tropism is composed of a transmembrane trimetric glycoprotein protruding from the viral surface. Structural and functional studies demonstrated that the spike protein the of coronaviruses can bind to angiotensin converting enzyme 2 (ACE2), a functional receptor for SARS-CoV. ACE2 expression is high in lung (high expression on lung epithelial cells), heart, ileum, and kidney. The lungs of severe COVID-19 patients demonstrate infiltration of a large number of inflammatory cells. Due to high ACE2 expression on the apical side of lung epithelial cells in the alveolar space, SARS-CoV-2 (COVID-19) can enter and destroy lung epithelial cells. Significant ACE2 expression on innate lymphoid cells (ILC)2, ILC3, and endothelial cells is also demonstrated. Pulmonary endothelial cells represent one third of the lung cells. Endothelial function includes promotion of anti-aggregation, fibrinolysis, and vasodilatation. Due to a significant role playing in thrombotic regulation, hypercoagulable profiles that are demonstrated in severe COVID-19 patients likely suggest significant endothelial injury. Pulmonary thrombosis and embolism accompanying elevation of d-dimer and fibrinogen levels have been demonstrated in severe COVID-19. In conclusion, whether these histopathological lesions are direct consequences of sepsis, SARS-CoV-2 (C)OVID-19), and /or multiple organ failure is difficult to conclude. Further studies on understanding the roles of ILC1, ILC2, ILC3, including the difference in response to SARS-CoV-2 (COVID-19) infection between children and adults are urgently needed to develop efficient targeted therapies.
\end{abstract}

Keywords: COVID-19, host cells, invasion, pathogenesis, pulmonary, pathology, SARSCoV-2
Volume 7 Issue 3 - 2020

\author{
Attapon Cheepsattayakorn, ${ }^{1,2,3}$ Ruangrong \\ Cheepsattayakorn ${ }^{4}$ \\ 1 I $0^{\text {th }}$ Zonal Tuberculosis and Chest Disease Center,Thailand \\ ${ }^{2}$ Faculty of Medicine, Western University, Thailand \\ ${ }^{3}$ Faculty of Public Health, St. Theresa International College, \\ Thailand \\ ${ }^{4}$ Department of Pathology, Faculty of Medicine, Chiang Mai \\ University, Thailand
}

Correspondence: Attapon Cheepsattayakorn, I0th Zonal Tuberculosis and Chest Disease Center, 143 Sridornchai Road Changklan Muang Chiang Mai 50100 Thailand, Tel 6653 |40767, 6653 276364, Fax 6653 I40773, 6653 273590,Email Attapon198@gmail.com

Received: September 10, 2020 | Published: November 04, 2020
Abbreviations: ACE2, angiotensin converting enzyme 2; $\mathrm{ACP}$, antigen presenting cell; AKI, acute kidney injury; ARDS, acute respiratory distress syndrome; COVID-19, coronavirus disease 2019; CRS, cytokine release syndrome; CT, computed tomography; DC, dendritic cell; DC-SIGN, dendritic-cell specific intercellular adhesion molecule-3-grabbing nonintegrin, DC-SIGNR; L-SIGN, DC-signrelated protein; EAE, experimental autoimmune encephalomyelitis; ESR, erythrocyte sedimentation rate; FGF, fibroblast growth factor; G-CSF, granulocyte-colony-stimulating factor; GM-CSF, granulocytemacrophage colony-stimulating factor; ICU, intensive care unit; IFN, interferon; IL, interleukin; ILC, innate lymphoid cell; IP, interferongamma-induced protein; MCP, monocyte chemoattractant protein; MERS-CoV, middle-east-respiratory-syndrome coronavirus; MIP, macrophage inflammatory protein; NK, natural killer; PD-1, programmed cell death-1; PDGF, platelet-derived growth factor; RNA, ribonucleic acid; SARS, severe acute respiratory syndrome; SARS-CoV, severe acute respiratory syndrome coronavirus; SARSCoV-2, severe acute respiratory syndrome coronavirus type 2; TCR, T cell receptor; Tim-3, T-cell Immunoglobulin and Mucin-3; TMPRSS2, transmembrane protease serine 2; TNF, tumor necrosis factor; VEGF, vascular endothelial growth factor

\section{Introduction}

Available data observed from China and India revealed that the individuals with age group of 20-50 year are likely to be infected by the SARS-CoV-2 (COVID-19). ${ }^{1,2}$ Singapore and Germany took measures by ramping up COVID-19 testing capacity quite early and by ensuring that all persons had equal opportunity to get tested, thus ensuring positive COVID-19 results early during COVID-19 progression. This meant that most cases were mild symptoms. ${ }^{3}$ South Korea constantly informed their people about the development of COVID-19 by using the a centralized messaging system and media. South Korea also used the Trace, Test, and Treat protocol to rapidly identify and isolate COVID-19 patients, while the United States limited this to severe COVID-19 patients with later broadening of this criterion as well as India and many European countries. South Korea also ensures free COVID-19 diagnostic testing through the universal healthcare, unlike the United States.

Currently, animal-to-human transmission of SARS-CoV-2 (COVID-19) has not yet been confirmed, whereas the main mode of transmission is human-to-human. Droplets are the main route of human-to-human transmission, whereas aerosols could be another 
route $^{4}$ in addition to stool-based transmission. ${ }^{5,6}$ Currently, no evidence is available to indicate intrauterine vertical transmission of SARSCoV-2 (COVID-19) in pregnant women. ${ }^{7}$ The Indian government concerns on how to identify and contain asymptomatic SARSCoV-2 (COVID-19) carriers, who could account for approximately $80 \%$ of COIVID-19-infected individuals. ${ }^{8}$ As all of SARS-CoV-2 (COVID-19) may not develop the disease, asymptomatic SARSCoV-2 (COVID-19)-infected carriers are at major risk of being superinfector with COVID-19. ${ }^{9}$ Some investigators hypothesize that a warm climate could reduce transmission by preventing SARS-CoV-2 (COVID-19) from surviving for longer periods of time on surfaces.

\section{Mechanism of SARS-CoV-2 (COVID-19) invasion into host cells}

Coronaviruses are enveloped and single-stranded ribonucleic acid (RNA) viruses of approximately $30 \mathrm{~kb}$ with infections of various host species. ${ }^{10}$ SARS-CoV-2 (COVID-19) are divided into four genera; $\alpha, \beta, \gamma$, and $\delta$ based on their genomic structure. Alpha and beta coronaviruses infect only mammals. ${ }^{11}$ SARS-CoV-2 (COVID-19), SARS-CoV, and Middle-East-Respiratory-Syndrome coronavirus (MERS-CoV) are classified to $\beta$ coronaviruses.

In the host, the life cycle of coronavirus consists of 5 steps: 1) attachment, 2) penetration, 3) biosynthesis, 4) maturation, and 5) release. Once viruses bind to host receptors (attachment), they enter host cells, particularly type II pneumocytes via endocytosis or membrane fusion (penetration). Once viral contents are released inside the host cells, viral RNA enters the host's nucleus for replication and making viral proteins (biosynthesis). New viral particles are produced (maturation) and released.

Coronaviruses consist of four structural proteins; spike (S), membrane (M), envelop (E), and nucleocapsid (N). ${ }^{12}$ Spike protein of coronaviruses which determines the diversity of coronaviruses and host tropism is composed of a transmembrane trimetric glycoprotein protruding from the viral surface. Spike protein comprises two functional subunits; S1 subunit is responsible for binding to the host cell receptor and S2 subunit is responsible for the fusion of the viral and cellular membranes. Structural and functional studies demonstrated that the spike protein the of coronaviruses can bind to angiotensin converting enzyme 2 (ACE2), ${ }^{13-15}$ a functional receptor for SARS-CoV. ${ }^{16}$ ACE2 expression is high in lung (high expression on lung epithelial cells), heart, ileum, and kidney. ${ }^{17}$ Further studies are needed for additional SARS-CoV-2 (COVID-19) binding targets.

After binding of SARS-CoV-2 (COVID-19) to the host protein, protease cleavage is underwent by the spike protein. Activation of the spike protein of SARS-CoV-2 (COVID-19) and MERS-CoV as a two-step sequential protein cleavage has been proposed as a model that consists of cleavage at the S1/S2 cleavage site for priming and a cleavage for activation at a position adjacent to a fusion peptide within the S2 subunit "S2" site. ${ }^{18-20}$ Following the cleavage at the S1/S2 cleavage site, $\mathrm{S} 1$ and $\mathrm{S} 2$ subunits remain non-covalently bound and the distal S1 subunit leads to the stabilization of the membrane-anchored S2 subunit at the pre-fusion state. ${ }^{14}$ Presumably activation of the spike protein for membrane fusion through irreversible and conformational changes is due to subsequent cleavage at the S2 site. ${ }^{21}$ Existence of furin cleavage site ("RPPA" sequence) at the $\mathrm{S} 1 / \mathrm{S} 2$ site is the unique characteristics of SARS-CoV-2 (COVID-19) among coronaviruses. During biosynthesis, the S1/S2 site of SARS-CoV-2 (COVID-19) is entirely subjected to cleavage in a drastic contrast to SARS-CoV spike protein that is incorporated without cleavage. ${ }^{14}$ The expression of furin makes SARS-CoV-2 (COVID-19) very pathogenic although the $\mathrm{S} 1 / \mathrm{S} 2$ site is also subjected to cleavage by other protease, such as cathepsin $\mathrm{L}$ and transmembrane protease serine 2 (TMPRSS2). ${ }^{20,22}$

T-cell mediated responses against coronaviruses are antigen presentation through dendritic cells (DCs) and macrophage that can phagocytize virus-infected-apoptotic epithelial cells contributing to antigen presentation to T cells. The expression of ACE2 on (splenic) dendritic cells and pulmonary alveolar macrophages is present but limited, based on the Immunological Genome database (http://rstats. immgen.org). DCs and macrophages may be primarily infected with virus. SARS-CoV-2 (COVID-19) uses another protein to bind to antigen presenting cells (ACPs) or not should be investigated. These ACPs move to the draining lymph nodes to present viral antigens to $T$ cells. In addition to ACE2, SARS-CoV can also bind to dendritic-cell specific intercellular adhesion molecule-3-grabbing nonintegrin (DCSIGN, high expression on dendritic cells and macrophages) and DCSIGN-related protein (DC-SIGNR, L-SIGN) ${ }^{23-25} \mathrm{CD} 8+\mathrm{T}$ cells kill viral infected cells, whereas CD4+ T cells activate B cells to promote the virus-specific antibody production.

Patients with severe COVID-19 demonstrated lymphopenia, especially in peripheral blood $\mathrm{T}$ cell $\mathrm{s}^{26,27}$ and increased plasma concentrations of granulocyte-colony stimulating factor (G-CSF), interleukin (IL)-6, IL-10, macrophage inflammatory protein (MIP)$1 \alpha$, monocyte chemoattractant protein 1 (MCP-1), and tumor necrosis factor (TNF)- $\alpha .{ }^{26-28}$ The higher levels of IL-6 are, the more severe conditions the COVID-19 patients are in. Higher expression of CD38, CD44, and CD69 is demonstrated in COVID-19 patients with activation of $\mathrm{CD} 4+$ and $\mathrm{CD} 8+\mathrm{T}$ cells. T cells exhaustion that could have led to the progression of COVID-19 is indicated by higher percentage of checkpoint receptor Tim-3+ PD-1+ subsets in CD4+ and CD8+ T cells. Another marker for T cells exhaustion is elevation of NK group 2 member A (NKG2A) on CD8+ T cells. ${ }^{29}$ Aberrant pathogenic $\mathrm{CD} 4+\mathrm{T}$ cells with co-expressing interferon (IFN) $-\gamma$ and granulocyte-macrophage colony-stimulating factor (GMCSF) are demonstrated in severe COVID-19 patients. ${ }^{26}$ Significant decrease in circulating $\mathrm{T}$ cells, the majority of infiltrating adaptive immune cells are primary cytotoxic CD8 $+\mathrm{T}$ cells. CD4 $+\mathrm{T}$ cells are also pathological cytotoxic $\mathrm{T}$ cells found in severe COVID-19 patients $^{30}$ with lung injury. ${ }^{31}$ These pathological CD4+ T cells release circulating monocytes responding to GM-CSF. Significant higher percentage of $\mathrm{CD} 14+\mathrm{CD} 16+$ inflammatory subsets are also identified in COVID-19 patients, but they are seldom exist in health individuals. These inflammatory CD14+CD16+ inflammatory monocytes demonstrate high IL-6 expression, that accelerates the progression of systemic inflammatory response. GM-CSF, a response to virus infection can assist in differentiation of innate immune cells augment $\mathrm{T}$ cell function, but GM-CSF can trigger tissue damage at excess. 32,33 Previous experimental autoimmune encephalomyelitis (EAE) models in adults revealed that GM-CSF $+\mathrm{IFN}-\gamma+\mathrm{CD} 4+\mathrm{T}$ cells were demonstrated upon strong $\mathrm{T}$ cell receptor (TCR) responses, whereas CD8+ T cells expressing GM-CSF were identified at higher percentage and secreted IL-6. Neutrophils, the majority of innate immune cells can induce lung injury. ${ }^{34-36}$

In addition to IL- 6 production, SARS-CoV-infected lung epithelial cells produce IL-8, a well-known chemoattractant for neutrophils and $\mathrm{T}$ cells. ${ }^{37}$ The three main components for innate immunity in human airway are epithelial cells, pulmonary alveolar macrophages, and dendritic cells (DCs), whereas DCs are located underneath the epithelium and macrophages reside at the apical side of the epithelium. ${ }^{37}$ The lungs of severe COVID-19 patients demonstrate 
infiltration of a large number of inflammatory cells. ${ }^{38,39}$ Due to high ACE2 expression on the apical side of lung epithelial cells in the alveolar space, ${ }^{40,41}$ SARS-CoV-2 (COVID-19) can enter and destroy lung epithelial cells. Significant ACE2 expression on innate lymphoid cells (ILC) 2, ILC $3,{ }^{42}$ and endothelial cells ${ }^{43,44}$ is also demonstrated. NK cells, a member of ILC1 constitute a majority of pulmonary ILCs, approximately $95 \%$, whereas ILC2 and ILC3 are responsible for mucous homeostasis. Nevertheless, there is a very limited knowledge of ILC2- and ILC3-involved coronavirus infection. Pulmonary endothelial cells represent one third of the lung cells. ${ }^{45}$ Endothelial function includes promotion of anti-aggregation, fibrinolysis, and vasodilatation. ${ }^{46}$ Due to a significant role playing in thrombotic regulation, ${ }^{46}$ hypercoagulable profiles that are demonstrated in severe COVID-19 patients likely suggest significant endothelial injury. Pulmonary endothelial injury can facilitate viral invasion through abnormal microvascular permeability. Pulmonary thrombosis and embolism accompanying elevation of d-dimer and fibrinogen levels have been demonstrated in severe COVID-19. The clinical features of SARS-CoV-2-infected patients vary from minimal symptoms to severe respiratory failure with multiple organ failure, in addition to pulmonary thrombosis and embolism. Computed tomography (CT) of the chest in COVID-19 patients reveals the characteristic pulmonary ground glass opacification even in the asymptomatic patients. ${ }^{47}$

SARS-CoV-2 (COVID-19) causes lung parenchymal injury by interstitial lung and/or alveolar inflammation via directly binding to the pulmonary ACE-2 receptors resulting in pneumonitis and respiratory failure. ${ }^{48}$ The related cytokine release syndrome (CRS) that mainly developed by the IL- $6^{48}$ can exacerbate both lung parenchymal and microvascular inflammation contributing to refractory acute respiratory distress syndrome (ARDS). ${ }^{49-51}$ Cardiac dysfunction with related pulmonary edema, such as cardiac arrhythmias, stress cardiomyopathy, myocarditis, etc. can be contributed by SARSCoV-2 (COVID-19) has been demonstrated by several previous studies. ${ }^{52-58}$ Severe brain injury in COVID-19 could induce neurogenic pulmonary edema due to the catecholamine storm. ${ }^{58}$ The incidence of acute kidney injury (AKI) among hospitalized COVID-19 patients is approximately $11 \%$, whereas actual AKI incidence in intensive care unit (ICU) remains uncertain. ${ }^{59}$ Proteinuria during SARCoV-2 (C)OVID-19) infection is reported approximately $7 \%$ to $63 \%$ of cases, ${ }^{60-63}$ reflecting diffuse acute renal tubular injury. ${ }^{64}$

$\mathrm{Su}$ et al. studied renal histopathological lesions in 26 dead COVID-19 patients and revealed that underlying conditions, such as diabetes, arteriosclerosis in patients with cardiovascular diseases and/ or ischemic renal glomeruli with hypertension. They found diffuse acute proximal tubular injury with cytoplasmic vacuoles that may associated with direct SARS-CoV-2 (COVID-19) in the podocytes with secondary foot process effacement and detachment of podocytes from the glomerular basement membrane and in the cytoplasm of the proximal tubular epithelium with expression of SARS-CoV nucleoprotein. Glomerular ischemia with fibrin thrombi within the glomerular capillary loops reflecting coagulation activation. Diffuse erythrocyte aggregation and obstruction in the lumen of peritubular and glomerular capillaries without red blood cell fragments, thrombi, fibrinoid, or platelet necrosis. ${ }^{65}$

Rhabdomyolysis has been noted in COVID-19 patients. ${ }^{66}$ The kidneys showed no evidence of complement activation. ${ }^{67}$ Hence, whether these histopathological lesions are direct consequences of sepsis, SARS-CoV-2 (C)OVID-19), and /or multiple organ failure is difficult to conclude ${ }^{68}$ Direct viral impact, liver congestion abnormalities, hypoxia-induced damage, and systemic inflammation are factors that are associated with COVID-19-associated liver damage.$^{69}$ The neurological symptoms are characterized as the centralnervous-system symptoms in severe cases, such as ataxia, epilepsy, dizziness, reduced consciousness, head pain, and acute cerebrovascular diseases ${ }^{70}$ and as the peripheral nervous system symptoms, such as fatigue, myalgia, neuralgia, hypoplasia, hypogeusia, and anosmia (or hyposmia). ${ }^{71-73}$ The pathogenesis of SARS-CoV-2 (COVID-19) in the nervous system is associated with direct viral attachment of the nerve endings in the peripheral nervous system or indirect injuries associated with the viral sepsis and the cytokine storm. ${ }^{74}$

The difference of pathophysiology between children and adults in COVID-19 is hypothesized as the following: 1) The expression level of ACE2 may differ between children and adults, ${ }^{41}$ 2) Children have a qualitatively different response to the SARS-CoV-2 (COVID-19) virus to adults, ${ }^{75}$ and 3 ) The simultaneous presence of other viruses in the mucosa of lungs and airways that are common in young children can contribute to SARS-CoV-2 (COVID-19) virus compete with them and limit its growth. ${ }^{76}$

\section{Conclusion}

Some questions involving SARS-CoV-2 (COVID-19) are needed to be answered include: 1) Do asymptomatic persons develop the disease at any point in time at all?, 2) How long do the patients shed the virus for ?, 3) Do the patients eventually develop antibodies ?, and 4) Is SARS-CoV-2 (COVID-19) stored in any individuals' tissue in a dormant state ? Additionally, further studies on understanding the roles of ILC1, ILC2, ILC3, including the difference in response to SARS-CoV-2 (COVID-19) infection between children and adults are urgently needed to develop efficient targeted therapies.

\section{Authors contributions}

Dr. Attapon Cheepsattayakorn conducted the study framework and wrote the manuscript. Associate Professor Dr. Ruangrong Cheepsattayakorn contributed to scientific content and assistance in manuscript writing. Both authors read and approved the final version of the manuscript.

\section{Competing interests}

The authors declare that they have no actual or potential competing financial interests.

\section{Funding sources}

The authors disclose no funding sources.

\section{References}

1. $83 \%$ percent of India's coronavirus patients are below the age of 50 : Health Ministry data. India Today; 2020.

2. https://econimictimes.indiatimes.com/news/politics-andnation/42-of-coronavirus-patients-in-21-40-age-bracket-govt/ articleshow/74987254.cms

3. Stafford N. COVID-19: why Germany's case fatality rate seems so low. British Medical Journal. 2020;7:369:m1395.

4. World Health Organization (WHO). Questions and answers on coronaviruses. Switzerland, Geneva: World Health Organization; 2020.

5. Holshue ML, DeBolt C, Lindquist S, et al. First case of 2019-novel coronavirus in the United States. N Engl J Med. 2020;382(10):929-936.

6. Yeo C, Kaushal S, Yeo D. Enteric involvement of coronavirus: is fecaloral transmission of SARS-CoV-2 possible? Lancet Gastroenterol Hepatol. 2020;5(4):335-337. 
7. Chen H, Guo J, Wang C, et al. Clinical characteristics and intrauterine vertical transmission potential of COVID-19 infection in nine pregnant women: a retrospective review of medical records. Lancet. 2020;395(10226):809-815.

8. Coronavirus pandemic: $80 \%$ of COVID-19 cases either asymptomatic or show mild symptoms. Health ministry-Moneycontrol.com. 2020.

9. Rothe C, Schunk M, Sothmann P, et al. Transmission of 2019-nCoV infection from an asymptomatic contact in Germany. $N$ Engl J Med. 2020;382(10):970-971

10. Channappanavar R, Zhao J, Perlman S. T cell-mediated immune response to respiratory corona viruses. Immunol Res. 2014;59(1-3):118-128.

11. Rabi FA, Al-Zoubi MS, Kasasbeh GA, et al. SARS-CoV-2 and Coronavirus disease 2019: what we know so far. J Virol. 2020;9(3):231.

12. Bosch BJ, der Zee van, de Haan CA, et al. The Coronavirus spike protein is a class I virus fusion protein :structural and functional characterization of the fusion core complex. J Virol. 2003;77(16):8801-8811.

13. Chen Y, Guo Y, Pan Y, et al. Structural analysis of the receptor binding of 2019-nCoV. Biochem Biophys Res Commun. 2009;525(1):135-140.

14. Walls AC, Park YJ, Tortorici MA, et al. Structure, function, and antigenicity of the SARS-CoV-2 spike glycoprotein. Cell. 2020;181(2):281-292.

15. Letko M, Marzi V, Munster V. Functional assessment of cell entry and receptor usage for SARS-CoV-2 and other lineage B betacoronaviruses. Nat Microbiol. 2020;5(4):562-569.

16. Li W, Moore MJ, Vasilieva N, et al. Angiotensin converting enzyme 2 is a functional receptor for the SARS coronavirus. Nature. 2003;426(6965):450-454.

17. Zou X, Chen K, Zou J, et al. Single-cell RNA-seq data analysis on the receptor ACE2 expression reveals the potential risk of different human organs vulnerable to 2019-nCoV infection. Front Med. 2020;14(2):185192.

18. Belouzard S, Chu VC, Whittaker GR. Activation of the SARS Coronavirus spike protein via sequential proteolytic cleavage at two distinct sites. Proc Natl Acad Sci U S A . 2009;106(14):5871-5876.

19. Millet JK, Whittaker GR. Host cell entry of Middle-East-RespiratorySyndrome Coronavirus after two-step, furin-mediated activation of the spike protein. Proc Natl Acad Sci U S A. 2014;111(42):15214-15219.

20. Ou X, Liu Y, Lei X, et al. Characterization of spike glycoprotein of SARSCoV-2 on virus entry and its immune cross-reactivity with SARS-CoV. Nat Commun. 2020;11(1):1620.

21. Belouzard S, Millet JK, Licitra BN, et al. Mechanisms of coronavirus cell entry mediated by the viral spike protein. Viruses. 2012;4(6):1011-1033.

22. Hoffmann M, Kleine-Weber H, Schroeder S, et al. SARS-CoV-2 cell entry depends on ACE2 and TMPRSS2 and is blocked by a clinically proven protease inhibitor. Cell. 2020;181(2):271-280.

23. Jeffers SA, Tusell SM, Gillim-Ross L, et al. CD209L(L-SIGN) is a receptor for severe acute respiratory syndrome coronavirus. Proc Natl Acad Sci U S A. 2004;101(44):15748-15753.

24. MarziA, Gramberg T, Simmons G, et al. DC-SIGN andDC-SIGNR interact with the glycoprotein of Marburg virus and the $\mathrm{S}$ protein of severe acute respiratory syndrome coronavirus. J Virol. 2004;78(21):12090-12095.

25. Yang ZY, Huang Y, Ganesh L, et al. pH-dependent entry of severe acute respiratory syndrome coronavirus is mediated by the spike glycoprotein and enhanced by dendritic cell transfer through DC-SIGN. J Virol. 2004;78(11):5642-5650.

26. Zhou Y, Fu B, Zheng X, et al. Pathogenic T cells and inflammatory monocytes incite inflammatory storm in severe COVID-19 patients. Natl Sci Rev. 2020.
27. Qin C, Zhou L, Hu Z, et al. Dysregulation of immune response in patients with COVID-19 in Wuhan, China. Clin Infect Dis. 2020;71(15):762-768.

28. Huang C, Wang Y, Li X, et al. Clinical features of patients infected with 2019 novel coronavirus in Wuhan, China. Lancet. 2020;395(10223):497-506.

29. Zheng M, Gao Y, Wang G, et al. Functional exhaustion of antiviral lymphocytes in COVID-19 patients. Cell Mol Immunol. 2020;17(5):533-535.

30. Fang M, Siciliano NA, Hersperker AR, et al. Perforin-dependent CD4+ T-cell cytotoxic contributes to control a murine poxvirus infection. Proc Natl Acad Sci U S A. 2012;109(25):9983-9988.

31. Small BA, Dressel SA, Lawrence CW, et al. CD8(+) T cell-mediated injury in vivo progresses in the absence of effector T cells. $J$ Exp Med. 2001;194(12):1835-1846.

32. Huang H, Wang S, Jiang T, et al. High levels of circulating GM-CSF $(+)$ CD4(+)T cells are predictive of poor outcomes in sepsis patients :a prospective cohort study. Cell Mol Immunol. 2019;16(6):602-610.

33. Croxford AL, Lanzinger M, Hartmann FJ, et al. The cytokine GM-CSF drives the inflammatory signature of CCR2+ monocytes and licenses autoimmunity. Immunity. 2015;43(3):502-514.

34. Young RE, Thompson RD, Larbi KY, et al. Neutrophil elastase(NE)deficient mice demonstrate a nonredundant role for $\mathrm{NE}$ in neutrophil migration, generation of proinflammatory mediators, and phagocytosis in response to zymosan particles in vivo. J Immunol. 2004;172(7):4493-4502.

35. Liu S, Su X, Pan P, et al. Neutrophil extracellular traps are indirectly triggered by lipopolysaccharide and contribute to acute lung injury. Sci Rep. 2016;6:37252.

36. Koutsogiannaki S, Shimaoka M, Yuki K. The use of volatile anesthetics as sedatives for acute respiratory distress syndrome. Transl Perioper Pain Med. 2019;6(2):27-38.

37. Yoshikawa T, Hill T, Li K, et al. Severe acute respiratory syndrome(SARS) coronavirus-induced lung epithelial cytokines exacerbate SARS pathogenesis by modulating intrinsic function of monocyte-derived macrophages and dendritic cells. J Virol. 2009;83(7):3039-3048.

38. Xu Z, Shi L, Wang Y, et al. Pathological findings of COVID-19 associated with acute respiratory distress syndrome. Lancet of Respir Med. 2020;8(4):420-422.

39. Tian S, Hu W, Niu L, et al. Pulmonary pathology of early-phase 2019 novel coronavirus(COVID-19) pneumonia in two patients with lung cancer. J Thorac Oncol. 2020;15(5):700-704.

40. Hamming I, Timens W, Bulthuis ML, et al. Tissue distribution of ACE2 protein, the functional receptor for SARS coronavirus :a first step in understanding SARS pathogenesis. J Pathol. 2004;203(2):631-637.

41. Jia HP, Look DC, Shi L, et al. ACE2 receptor expression and severe acute respiratory syndrome coronavirus infection depend on differentiation of human airway epithelia. J Virol. 2005;79(23):14614-14621.

42. Yuki K, Jujiogi M, Koutsogiannaki S. COVID-19 pathophysiology : a review. Clinical Immunology. 2020;215:108427.

43. Lovren F, Pan Y, Quan A, et al. Angiotensin converting enzyme-2 confers endothelial protection and attenuates atherosclerosis. Am Physiol Heart Circ Physiol. 2008;295(4):H1377-H1384.

44. Sluimer JC, Gasc JM, Hamming I, et al. Angiotensin converting enzyme-2 (ACE2) expression and activity in human carotid atherosclerotic lesions. $J$ Pathol. 2008;215(3):273-279.

45. Zeng H, Pappas C, Belser JA, et al. Human pulmonary microvascular endothelial cells support productive replication of highly pathogenic avian influenza viruses : possible involvement in the pathogenesis of human H5N1 virus infection. J Virol. 2012;86:(2):667-678. 
46. Wang $\mathrm{M}$, Hao $\mathrm{H}$, Leeper $\mathrm{NJ}$, et al. Thrombotic regulation from the endothelial cell perspectives. Arterioscler Thromb Vasc Biol. 2018;38(6):e90-e95.

47. Guan WJ, Ni ZY, Hu Y, et al. Clinical characteristics of coronavirus disease 2019 in China. N Engl J Med. 2020;382(18):1708-1720.

48. Alharthy A, Faqihi F, Memish ZA, et al. Lung injury in COVID-19; an emerging hypothesis. ACS Chemical Neuroscience. 2020;11:2156-2158.

49. Tnag N, Bai H, Chen X, et al. Anticoagulant treatment is associated with decreased mortality in severe coronavirus disease 2019 patients with coagulopathy. J Thromb Haemostasis. 2020;18:1094-1099.

50. Klok FA, Kruip MJHA, van der Meer NJM, et al. Incidence of thrombotic complications in critically ill ICU patients with COVID-19. Thromb Res. 2020;191:145-147.

51. Zhang Y, Xiao M, Zhang S, et al. Coagulopathy and antiphospholipid antibodies in patients with COVID-19. N Engl J Med. 2020;382(17):e38.

52. Nguyen D, Nguyen T, De Bels D, et al. A case of Takotsubo cardiomyopathy with COVID-19. Eur Heart J: Cardiovasc Imaging. 2020;21(9):1052.

53. Roca E, Lombardi C, Campana M, et al. Takotsubo syndrome associated with COVID-19. J Case Rep Intern Med. 2020;7(5):001665.

54. Meyer P, Degrauwe S, Van Delden C, et al. Typical takotsubo syndrome triggered by SARS-CoV-2 infection. Eur Heart J. 2020;41:(19):1860.

55. Minhas AS, Scheel P, Garibaldi B, et al. Takotsubo syndrome in the setting of COVID-19 infection. JACC Case Rep. 2020;2:1321.

56. Sala S, Peretto G, Gramegna M, et al. Acute myocarditis presenting as a reverse Takotsubo syndrome in a patient with SARS-CoV-2 respiratory infection. Eur Heart J. 2020;41(19):1861-1862.

57. Kochi AN, Tagliari AP, Forleo GB, et al. Cardiac and arrhythmic complications in patients with COVID-19. J Cardiovasc Electrophysiol. 2020;31(5):1003-1008

58. Inciardi RM, Lupi L, Zaccone $\mathrm{G}$, et al. Cardiac involvement in a patient with coronavirus disease 2019 (COVID-19). JAMA Cardiol. 2020;5(7):819-824

59. Gabarre P, Dumas G, Dupont T, et al. Acute kidney injury in critically ill patients with COVID-19. Intensive Care Med. 2020;46:1339-1348.

60. Wang L, Li X, Chen X, et al. Coronavirus disease 19 infection does not result in acute kidney injury: an analysis of 116 hospitalized patients from Wuhan, China. AJN. 2020;51(5):343-348.

61. Chen Y, Luo R, Wang K, et al. Kidney disease is associated with in-hospital death of patients with COVID-19. Kidney Int. 2020;97(5):829-838.

62. Anti-2019-nCoV Volunteers, Li Z, Wu M, et al. Caution on kidney dysfunctions of 2019-nCoV patients. medRxiv 2020.
63. Cao M, Zhang D, Wang Y, et al. Clinical features of patients infected with the 2019 novel coronavirus (COVID-19) in Shanghai, China medRxiv2020.03.04.20030395.

64. Martínez-Rojas MA, Vega-Vega O, Bobadilla NA. Is the kidney a target of SARS-CoV-2? American Journal of Physiology-Renal Physiology. Ajprenal00160.2020.

65. Su H, Yang M, Wan C, et al. Renal histopathological analysis of 26 postmortem findings of patients with COVID-19 in China. Kidney Int 2020; 98(1):219-227.

66. Jin M, Tong Q. Early release-rhabdomyolysis as potential late complication associated with COVID-19. Emerging Infectious Diseases. CDC 2020;26(7):2020.

67. McCullough JW, Renner B, Thurman JM. The role of the complement system in acute kidney injury. Semin Nephrol. 2013;33(6).

68. Lerolle N, Nochy D, Guérot E, et al. Histopathology of septic shock $\beta$ induced acute kidney injury: apoptosis and leukocytic infiltration. Intensive Care Med. 2010;36(3):471-478.

69. Chau TN, Lee KC, Yao H, et al. SARS-associated viral hepatitis caused by a novel coronavirus: report of three cases. Hepatology (Baltimore MD). 2004;39(2):302-310.

70. Mao, et al. Neurological manifestations of hospitalized patients with COVID-19 in Wuhan: a retrospective case series study, China. JAMA Neurol. 2020; 77(6):683-690.

71. De Corato A, Lisi L, Capuano A, et al. Trigeminal satellite cell express functional calcitonin gene-related peptide receptors, whose activation enhances interleukin-1 $\beta$ pro-inflammatory effects. $J$ Neuroimmunol. 2011;237(1-2):39-46.

72. Sun P, Lu X, Xu C, et al. Understanding of COVID-19 based on current evidence. J Med Virol. 2020.

73. Yang W, Cao Q, Qin L, et al. Clinical characteristics and imaging manifestations of the 2019 novel coronavirus disease (COVID-19): a multi-center study in Wenzhou city, Zhejiang, China. J Infect. 2020; 80(4):388-393.

74. Ferrari S, Vento S, Monaco S, et al. Human immunodeficiency virusassociated peripheral neuropathies. In: Mayo Clinic Proceedings. Volume 2. 2006; Elsevier. Pp. 213-219.

75. Saule P, Trauet J, Dutriez V, et al. Accumulation of memory T cells from childhood to old age: central and effector memory cells in CD4(+) versus effector memory and terminally differentiated memory cells in CD8(+) compartment. Mech Ageing Dev. 2006; 127(3): 274-281.

76. Nickbakhsh S, Mair C, Matthews L, et al. Virus-virus interactions impact the population dynamics of influenza and the common cold. Proc Natl Acad Sci U S A. 2019;116(52):27142-27150. 